\title{
From Zero to Hero, the rise of the Trauma and Orthopaedic discharge summary
}

Jonathan Evans, Alexander Armstrong

\begin{abstract}
Discharge summaries document key information after an in-patient care episode and hand back care to the GP. Locally our orthopaedic department used carbon-copy paper as a discharge document. Anecdotally, these were thought to be inaccurate, illegible and of poor quality. Complaints from GPs became a catalyst in assessing the accuracy and presenting it to the department.

A baseline dataset of inaccuracies was collected. This became a good starting point to develop a new improved system of discharging orthopaedic patients. We proposed to develop a bespoke, electronic, patient centred discharge document. Our aims were to improve patient safety, quality and develop an adaptable document, which could be updated to be in line with local CQUINs. The initial challenges of the resistance to change within the department were overcome by presenting data in an open forum with an agreed trial period.
\end{abstract}

A multidisciplinary team was formed, including managers, consultants, junior doctors, allied health professionals and IT personnel. This facilitated live decision making, streamlining the processes. We created an adaptable online document, which enabled regular updates. This inturn improved the overall accuracy of the document, improved prescription of key medications and ensured we were able to fulfil local CQUINs. We highly recommend the use of subspecialty specific electronic discharge summaries.

\section{Problem}

Locally, discharge summaries were hand-written on carbon-copy paper. Complaints had been received from GPs highlighting issues of legibility and quality as well as frequent requests for further information from the clinical coding team. Illegible handwriting was compounded by only a carbon copy being available in the patient notes.

Copies sent to the GP were subject to postal delays and often GPs would not expect to receive the document for days. In the acute phase post-operatively this could cause problems with GP follow up, as GPs did not have the necessary information in a timely way.

\section{Background}

Providing an accurate discharge document is one of the duties of a doctor set out by the General Medical Council (GMC) [1]. Discharge documents provide a summary of the in-patient stay, and transfer care from secondary to primary care.

In addition to passing information to primary care, the discharge summary is used to prescribe medications to take home. The discharge summary also serves as the principle source of information to Trust clinical coders. Coders are responsible for describing in-patient episodes and procedures to the finance department and subsequently commissioners. If the discharge document is inaccurate, coding errors result in incorrect charging of tariffs to the Clinical Commissioning Group (CCG).

\section{Baseline Measurement}

Two weeks of paper discharge summaries were evaluated. The discharge summary accuracy was compared to patients' hospital notes, looking at five key pieces of information that should be included on all discharge summaries. This information was derived from the Scottish Intercollegiate Guidelines Network Discharge Document [2]. VTE (venous thromboembolism) prophylaxis was included in the analysis because it is very important in particular for patients who have had orthopaedic surgery. The six pieces of information were agreed within the department and with the quality improvement team. This revealed inaccuracies in the information being passed to the GP, as follows:

Correct consultant. $82.6 \%$

Correct diagnosis. $80.7 \%$

Correct procedure. $81.7 \%$ (of 60 patients)

Complications/significant concurrent illnesses documented. $46.4 \%$ (of 13 patients)

Documentation of medication changes. $25.8 \%$ (of 31 patients)

Prescription of pharmacological VTE prophylaxis following hip and knee replacements $76 \%$

\section{Design}

We aimed to develop a bespoke, patient centred, electronically completed, template, to address not only the problems of inaccuracy on discharge summaries but also legibility, timeliness, incorrect tariff charging, and failure to prescribe important 
medication for the patient to take home.

A working group was formed within the Trauma and Orthopaedic department to agree and implement changes to the discharge summary. The team analysed each problematic area using using PDSA methodology whilst reacting accordingly. This group was truly multidisciplinary with representatives from junior doctors, consultant surgeons, consultant ortho-geriatricians, pharmacists, clinical coders and the information-technology department forming the core, with others providing information when required.

A new bespoke Trauma and Orthopaedic electronic discharge proforma was agreed upon using information gleaned from these PDSA cycles, and once agreed, uploaded to the online system.

A formal teaching induction conducted by IT ensured all doctors became familiar with the new online system.

Regular meetings of the working group and re-assessment of progress as well as a close relationship with the IT department enabled 'live' decision making to address any 'teething' problems identified with the process.

\section{Strategy}

PDSA cycle 1: Accuracy of information

The baseline measurement showed inaccuracy in simple information such as patient demographics, responsible consultant and information regarding diagnosis and operations. By creating an interface with other hospital systems many of these areas "autopopulated" therefore removing an opportunity for error or omission. The accuracy of electronic discharge summaries after the change was compared to the paper documents. This demonstrated an improvement in accuracy in all of the parameters measured.

PDSA cycle 2: Bone protection

Assessment of a patients requirement for pharmacological bone protection assessment is essential in fracture prevention in orthogeriatric patients and also part of a local CQUIN. The prescription of bone protection was identified as area of weakness in initial review of accuracy. We knew that all patients were being assessed by an ortho-geriatrician as to their bone-protection requirements however it seemed the information was not making its way onto the discharge summary. A mandatory section box was incorporated into the electronic template ensuring the assessment was documented clearly and if required, bone protection was prescribed and highlighted any outstanding investigations (e.g.DEXA scans) to the GP. This immediately increased the documentation of the assessment but also served as a reminder in the rare patient that had not had an assessment

PDSA cycle 3: Pharmacological VTE prophylaxis

VTE following hip and knee replacement surgery carry significant morbidity and mortality [3]. According to both NICE and local guidelines, all patients undergoing hip and knee arthroplasty surgery should be discharged with (approximately) six weeks of pharmacological VTE prophylaxis [4]. A review of adherence to this by reviewing paper discharge summaries over one month, focusing on arthroplasty patients, revealed only $76 \%$ (71/93) of patients were actually receiving this. The provision of a discreet area for the prescription of pharmacological VTE prophylaxis seemed to work as a reminder as following its implementation $100 \%$ of patients were assessed for pharmacological VTE prophylaxis and $99 \%$ were discharged on the correct modality (according to local policy).

PDSA cycle 4: Co-morbidities

Patients with any significant past medical history (as defined by local commissioners) attract a higher-level tariff for operations or admissions. We looked at 44 trauma discharge summaries for the documentation of these specific co-morbidities. Of the 44 patients, we noted that $22(50 \%)$ had a co-morbidity meaning the higher-level tariff was payable that had not been documented on the discharge summary. This resulted in the standard tariff being charged by the Trust rather than the higher level. Clinical coders use the discharge summary as the main source of information for definition of the tariff and as such it is vital that co-morbidities are accurately documented on the summary. Review of these 44 patients with the Trust finance department revealed that if all 22 patients had been correctly coded the Trust would have received an additional $£ 14000$.

A "drop down box" containing all the manadatory co-morbidities has been included in the most recent version of the template however the results of this change are still to be seen.

\section{Post-Measurement}

As seen in the table below an improvement was seen in the overall accuracy. Our most impressive results came in the documentation of pharmacological bone protection assessment and VTE prophylaxis (following hip and knee replacement surgery).

A further interface between operating theatre computer systems and discharge summaries will, we hope, increase the accuracy further, in particular the specific nomenclature used for operations and procedures.

See supplementary file: ds2216.xls "20130917-Dis_sum_final_results"

\section{Lessons and Limitations}

Departmental changes are always a challenge and there were certainly some members of staff who were sceptical about our proposed changes. We advocate open forums to present and discuss any problems uncovered in your current practice. Trial periods evaluate success and cements confidence before making permanent changes. Forming a multidisciplinary working group that meets regularly and has the authority to make changes has facilitated real time decision making. The electronic document can be updated on an "as required" basis and we found this maximised the potential of the document. Flexibility enabled us to easily modify 
the document keeping in-line with local CQUIN requirements, which comes with significant financial incentive.

A limitation of this project was not having GP or member of the CCG as part of the MDT. We feel this would add significant value as well as helping to ensure we were providing the service and information that the CCG would like.

\section{Conclusion}

Electronic discharge summary template has improved accuracy, legibility and timeliness. Close relationships with IT services and the production of an interface between computer systems has ensured on-going incremental development.

The inclusion of discreet sections for vital information serves as a reminder to include this information and provides accurate information for clinical coders, which can have considerable financial implications. Bespoke specialty-specific electronic discharge summaries are highly recommended as we feel they increase accuracy, timeliness, safety and as such, overall quality.

\section{References}

1. Good medical practice (2013). http://www.gmc-uk.org/static documents/content/GMP_2013.pdf 51447599.pdf

2. Scottish Intercollegiate Guidelines Network (SIGN) Discharge Document. http://www.sign.ac.uk/pdf/sign128.pdf

3. Current guidelines for total joint VTE prophylaxis: dawn of a new day. Barrack RL. J Bone Joint Surg Br. 2012 Nov;94(11 Suppl A):3-7. doi: 10.1302/0301-620X.94B11.30824

4. NICE Guideline CG92

\section{Declaration of interests}

Jonathan Evans and Alexander Armstrong are joint first authors.

The are no conflicts of interest.

\section{Acknowledgements}

Paul Bowden and others at RD\&E IMT Shared Services,

Gaynor Hitchcock (Governance Support \& Implementation Lead

Critical Care / Trauma \& Orthopaedics RD\&E NHS FT)

John Charity, Associate Specialist in Trauma and Orthopaedics 